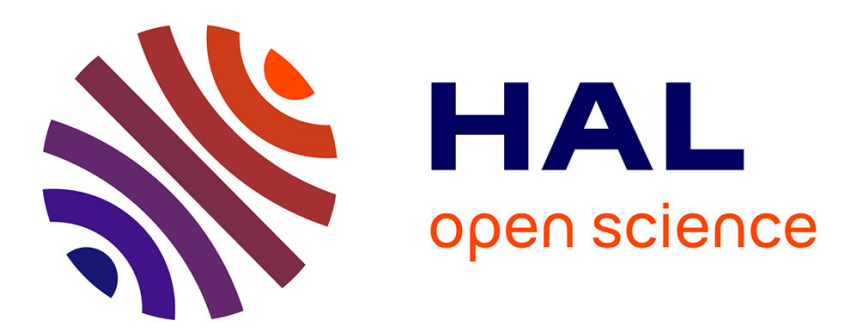

\title{
A Novel Angle-based Clustering Algorithm for Vehicular Ad Hoc Networks
}

Mohamed Hadded, Paul Mühlethaler, Anis Laouiti, Leila Azouz Saidane

\section{To cite this version:}

Mohamed Hadded, Paul Mühlethaler, Anis Laouiti, Leila Azouz Saidane. A Novel Angle-based Clustering Algorithm for Vehicular Ad Hoc Networks. IWVSC 2016, Aug 2016, Kuala Lumpur Malaysia. hal-01379221

\section{HAL Id: hal-01379221 \\ https://hal.science/hal-01379221}

Submitted on 11 Oct 2016

HAL is a multi-disciplinary open access archive for the deposit and dissemination of scientific research documents, whether they are published or not. The documents may come from teaching and research institutions in France or abroad, or from public or private research centers.
L'archive ouverte pluridisciplinaire $\mathbf{H A L}$, est destinée au dépôt et à la diffusion de documents scientifiques de niveau recherche, publiés ou non, émanant des établissements d'enseignement et de recherche français ou étrangers, des laboratoires publics ou privés. 


\title{
A Novel Angle-based Clustering Algorithm for Vehicular Ad Hoc Networks
}

\author{
Mohamed Hadded ${ }^{* \dagger}$, Paul Muhlethaler ${ }^{\dagger}$, Anis Laouiti* and Leila Azouz Saidane ${ }^{\ddagger}$ \\ *SAMOVAR, Télécom SudParis, CNRS, Université Paris-Saclay, 9 rue Charles Fourier 91011 EVRY, France \\ ${ }^{\dagger}$ INRIA, BP 105. 78153 Le Chesnay Cedex, Paris-Rocquencourt, France \\ ${ }^{\ddagger}$ RAMSIS Team, CRISTAL Laboratory, 2010 Campus University, Manouba, Tunisia \\ \{mohamed.hadded, anis.laouiti\}@ telecom-sudparis.eu, paul.muhlethaler@inria.fr, leila.saidane@ensi.rnu.tn
}

\begin{abstract}
A vehicular ad hoc network (VANET) is a mobile network in which vehicles acting as moving nodes communicate with each other through an ad hoc wireless network. VANETs have become the core component of Intelligent Transportation Systems (ITS) which aim to improve the road safety and efficiency. Only if the communication scheme used in a VANET is stable can these aims be achieved. Frequent changes in network topology and breaks in communication raise challenging issues in the design of communication protocols for such networks. Currently, clustering algorithms are being used as the control schemes to reduce changes in VANET topologies. However, the design of a clustering algorithm becomes a difficult task in VANETs when there are many road segments and intersections. In this work, we propose an Angle based Clustering Algorithm (ACA), which exploits the angular position and the direction of the vehicles to select the most stable vehicles that can act as cluster heads for a long period of time. The simulation results reveal that ACA significantly outperforms other clustering protocols in terms of cluster stability.
\end{abstract}

Keywords-VANET, Cluster Protocol, Ad hoc Networks, Mobility Direction, Angle.

\section{INTRODUCTION AND MOTIVATION}

Vehicle-to-Vehicle technology provides communication between vehicles through an ad hoc wireless network and eliminates the need for a central station to control the network topology. These vehicular ad hoc networks (VANETs) are characterized by the self-organization of the nodes and rapid changes in network topology due to the high speed of the vehicles. As breaks in communication links frequently occur in VANETs, ensuring communication stability is more difficult in VANETs than in standard MANETs. An effective and cheap solution to reduce the impact of mobility and improve the VANET network connectivity consists in establishing a hierarchical clustering structure within the network.

Designing of an efficient clustering protocol is no simple task in VANETs due to the rapid changes in network topology. Several VANET research studies in the literature have focused on developing clustering protocols, most of which use the mobility direction metric to form clusters. However, the mobility direction is not always sufficient to insure clustering stability in VANETs as can be seen in Figure 1 where the three vehicles $v_{1}, v_{2}$ and $v_{4}$ are considered to be moving in the same direction and thus these vehicles can be grouped together to form a cluster. Since vehicle $v_{4}$ and vehicles $v_{1}$ and $v_{2}$ are not moving on the same road, vehicle $v_{4}$ will leave the cluster after a short period and it will need to join a new cluster. In this paper, we present an Angle-based Clustering Algorithm (ACA), which uses the angle between velocity vectors of vehicles as a metric to form stable clusters. In ACA, two vehicles can form a cluster if and only if the angle between their velocity vectors is acute.

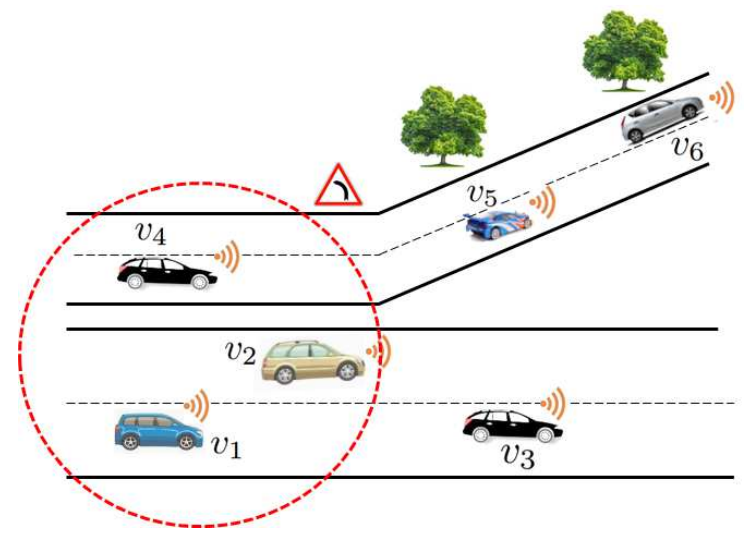

Fig. 1. Mobility direction based clustering.

\section{RELATED WORK}

Clustering is the best known method in VANETs to enable efficient resource allocation with low overhead and to reduce the relative mobility between neighboring vehicles. Several VANET research studies in the literature have focused on developing clustering protocols, most of which are based on MANET clustering techniques. Some of the most significant protocols are described below.

In [1], the authors proposed an Adaptive Weighted Clustering Protocol (AWCP) which is road map dependent and uses road IDs and movement direction in order to make the clusters' structure as stable as possible. However AWCP is based on the assumption that each vehicle is equipped with a digital mapping device, thus it can not operate in environments where vehicles without maps are present. In this work, we suppose that the vehicles are not equipped with digital road map devices and thus they can not obtain the road IDs on which they are traveling. In [2] the authors have proposed a multi-metric algorithm for cluster head elections, called Threshold-based Technique (TB), suitable for highway areas. In addition to the position and the direction, this algorithm uses a speed difference metric as a new parameter to increase the cluster lifetime. The vehicles that are moving at high speed are regrouped into one cluster, while the vehicles moving at low 
speed are grouped into another cluster. An Adaptable MobilityAware Clustering Algorithm based on Destination positions (AMACAD), is proposed and evaluated by Morales et al. [3]. The goal of this work is to develop a clustering protocol with an efficient message exchange mechanism, which improves cluster stability in VANETs. AMACAD performs clustering based upon information such as current location, vehicle velocity, relative destination and final destination of vehicles. A Multi-Head Clustering Algorithm, called Center-Position and Mobility (CPM), was proposed in [4]. This technique aims to create stable clusters and reduce re-clustering overhead by supporting single and multiple cluster heads. In the cluster head election phase, vehicles within communication range are organized into clusters and one vehicle for each cluster is elected to act as a Master Cluster Head (MCH). Then, some cluster members are selected to be Slave Cluster Heads (SCHs). In order to form stable clusters, the authors imposed that all the vehicles in a cluster are moving in the same direction.

Several other clustering algorithms designed for MANETs also work in VANETs and are frequently used for comparison with other VANET clustering protocols. For instance, the Lowest-ID clustering algorithm (LID) [5] is based on electing a node with the smallest ID as a cluster head. The HighestDegree algorithm (HD) [6] selects a node as a cluster head based on the nodes' connectivity. The node with the maximum number of neighbors becomes the cluster head. The Weighted Clustering Algorithm (WCA) [7] elects a node to act as a cluster head based on a combined weight which includes the number of its neighbors, their average distance and the node's average speed, and battery-life. MOBIC [8] is a Mobilitybased clustering algorithm designed for MANETs which is also used in VANETs. MOBIC is a mobility based version of the Lowest-ID algorithm and uses a signal power level metric to elect cluster heads. In this paper, we propose an angle-based clustering algorithm in which only the vehicles that are located on the same road segment and moving in the same direction can form a cluster by making an acute angle with the cluster heads and their members.

\section{Angle-BASEd Clustering Algorithm}

\section{A. Assumptions}

A VANET in a highway scenario consists of a set of vehicles moving in opposite directions and under varying traffic conditions (speed, density). ACA is based on the assumption that each vehicle in a VANET is equipped with a GPS (Global Positioning System) or a GALLILEO receiver that also allows it to obtain an accurate real-time three-dimensional geographic position (latitude, longitude and altitude), speed and exact time.

\section{B. Description}

ACA consists of three main phases: Stable neighbor detection, cluster head election and cluster maintenance.

1) Stable Neighbor detection: On the highway, vehicles traveling in the opposite direction to a reference cluster head will soon lose contact with it, but those traveling in the same direction will keep a relatively stable link state with the reference cluster head. So we should group the vehicles based on their mobility directions. In fact, the vehicles in n-road junctionz are grouped into $2 \times n$ different groups $\left(g_{1}, \ldots, g_{2 \times n}\right)$ according to their directions $\left(d_{1}, \ldots, d_{2 \times n}\right)$. Figure 2 shows an example of eight possible directions $\left(d_{1}, \ldots, d_{8}\right)$ of a 4 road junction. As shown in this figure, based on direction information, the vehicles can be grouped into eight different groups; each of which is characterized by one unit vector such as $(1,0),(0,1)$, etc. Two vehicles $v, w$ with velocity vectors $\left(v_{x}, v_{y}\right)$ and $\left(w_{x}, w_{y}\right)$ can be grouped together, if the angle between their velocity vectors is acute.
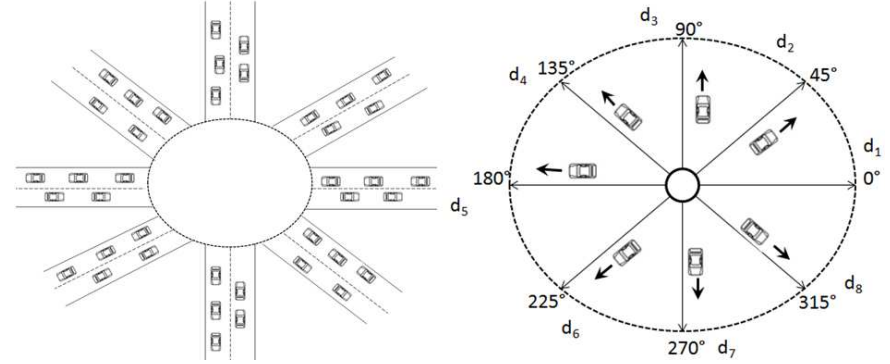

Fig. 2. The eight basic directions and their ranges at a 4-road junction.

As in [9], we can find whether two vehicles are moving in the same direction based on the angle $\theta$ between their velocity vectors. Let us suppose the position of two vehicles $v_{1}$ and $v_{2}$ at time $t$ are $\left(x_{1}, y_{1}\right),\left(x_{2}, y_{2}\right)$, and at time $t+\Delta t$ (where $\Delta t$ is a short time) are $\left(\hat{x_{1}}, \hat{y_{1}}\right),\left(\hat{x_{2}}, \hat{y_{2}}\right)$, respectively, as shown in Figure 3.

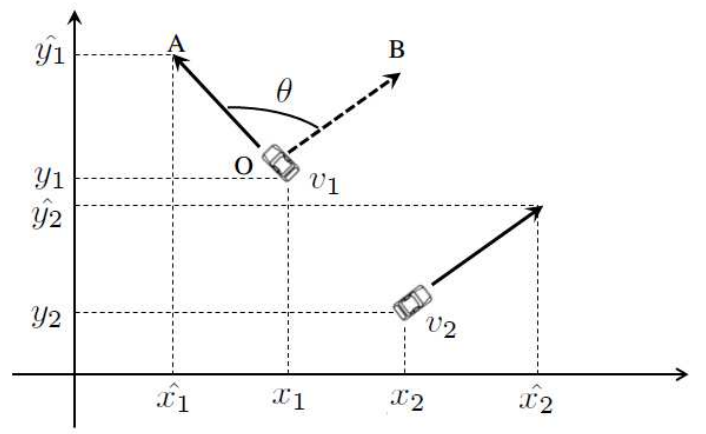

Fig. 3. Moving direction angle calculation.

The angle $\theta$ between two given velocity vectors is given by the following expression [10]:

$$
\overrightarrow{O A} \cdot \overrightarrow{O B}=\|\overrightarrow{O A}\| \times\|\overrightarrow{O B}\| \times \cos \theta
$$

$$
\theta=\arccos \left(\frac{\Delta x_{1} * \Delta x_{2}+\Delta y_{1} * \Delta y_{2}}{\sqrt{\Delta x_{1}^{2}+\Delta y_{1}^{2}} * \sqrt{\Delta x_{2}^{2}+\Delta y_{2}^{2}}}\right)
$$

$$
\left\{\begin{array}{l}
\Delta x=\hat{x}-x \\
\Delta y=\hat{y}-y
\end{array}\right.
$$

After receiving of a HELLO message from all each of its one-hop neighbors, vehicle $i$ only considers neighbors that 
have an angular directions equal to $\theta_{i} \pm \delta$, where $\theta_{i}$ is the angular direction of vehicle $i$ and $\delta$ is an angular value that represents the range of angles in which two vehicles are considered to be moving in the same direction. The authors in [9] propose that two velocity vectors are non-parallel if the smallest angle between the vectors is higher than $18^{\circ}$. Moreover, vehicle $i$ ignores all HELLO messages broadcasted from neighbors that have non-parallel velocity vectors. Therefore, the direction of the vehicles velocity vectors can help to build a stable cluster structure by grouping only the vehicles that have parallel velocity vectors in the same cluster, as shown in Figure 4.

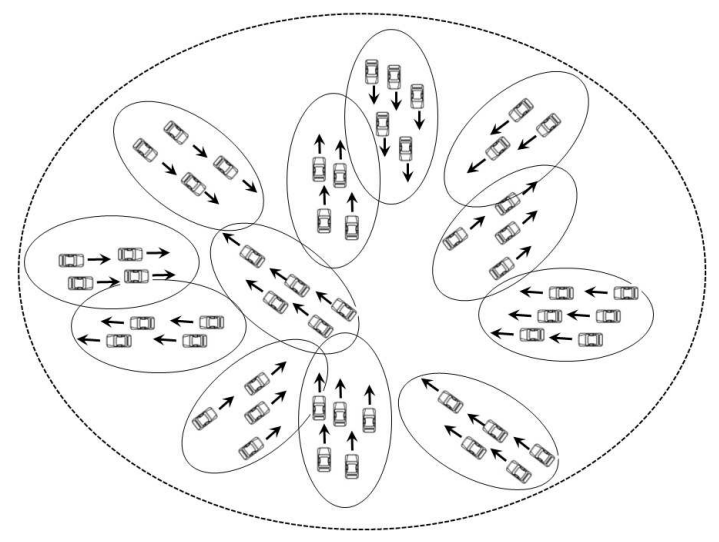

Fig. 4. Angle-based Clustering.

We propose an angle-based neighbor detection by exploiting the linear feature of a VANET network topology. Instead of discovering neighboring vehicles by exchanging Hello packets over the entire communication range, we have used an angular technique that allows each vehicle to identify the stable neighbors that it can form a cluster with and does not consider neighboring vehicles that are moving at an obtuse angle. This angular methodology helps us to build stable clusters and to reduce the overhead generated by the re-clustering mechanism due to the false merges at road intersections.

2) Cluster head election: In this work, we present our cluster head election algorithm based on the one defined in [11]. Initially, all vehicles are in the Undecided State (US). To divide the network into clusters, each active vehicle changes its state to Cluster Head Candidate (CHC) and it starts to broadcast a HELLO message periodically containing all the necessary information $\langle V I D$, old_position, current_position, speed $\rangle$ to its One-Hop neighbors $(\mathrm{OH})$. Each vehicle periodically updates its old_position and current_position every $100 \mathrm{~ms}$. On receiving HELLO messages from all its one-hop neighbors, each vehicle will calculate the angles between its velocity vector and those of its one-hop neighbors by using the position information received in the Hello messages. Each pair of vehicles can find whether they are moving in the same direction based on the angle value between their velocity vectors. In order to form stable clusters, only hello messages received from vehicles that are moving with an angle less than $\phi$ are considered, and the other messages are rejected. After that, each vehicle $i$ will update its One-Hop neighbor list $\left(O H_{i}\right)$ and it will calculate its current weight $\omega(i, t)$ using the following normalized weight function (2). This function consists of three parts, i.e., the average-distance to the direct neighbors (i), the average-speed (ii) and the number of neighbors (iii).

$$
\omega(i, t)=w_{1} * \frac{\delta(i, t)}{\tau}+w_{2} * \frac{|\nu(i, t)-\rho(i, t)|}{\vartheta}-w_{3} * \frac{n(i, t)}{\psi}
$$

Where $w_{1}, w_{2}$ and $w_{3}$ are the balancing factors such that $\sum_{k=1}^{3} w_{k}=1, \tau$ is the maximum radius of the vehicles, $\vartheta$ is the maximum allowed speed on the highway and $\psi$ is the cluster size. We note that the three weight factors are in conflict. For simplicity, we assume that all the factors should be minimized. In fact, the multiplication of the third weight factor by $(-1)$ allows us to transform a maximization to minimization. Then, each node $i$ broadcasts a beacon message containing all the necessary information for the $\mathrm{CH}$ election algorithm $\langle V I D, \omega, C H-I D\rangle$. Vehicle $i$ announces itself as a $\mathrm{CH}$ by assigning its own ID to the CH-ID field of the election beacon. When a vehicle $i$ receives beacons from its one-hop neighbors, it sorts its neighbor list $O H_{i}$ according to the weights received in the beacons, and then it executes the cluster head election algorithm to change its status from $\mathrm{CH}$ to Cluster Member (CM), Cluster Gateway (CG) or remain $\mathrm{CH}$.

$$
\omega(i, t)=\left\{\min \omega(j, t) \forall j \in O H_{i}\right\}(3), \quad n(i, t) \leq \psi
$$

The vehicle $i$ that satisfies the two properties (3) and (4) at instant $t$ is elected as the $\mathrm{CH}$. Then, all vehicles that are within transmission range of the $\mathrm{CH}$ become $\mathrm{CMs}$ or CGs and are not allowed to participate in another cluster head election procedure. The $\mathrm{CH}$ election algorithm terminates once all the vehicles become either a $\mathrm{CH}, \mathrm{CM}$ or a $\mathrm{CG}$.

3) Cluster maintenance: In VANETs, a vehicle can join or leave a cluster at any time. These two operations will have only local effects on the topology of the cluster if the vehicle is a $\mathrm{CM}$. However, if the vehicle is the $\mathrm{CH}$, it must hand over the responsibility to one of the very close cluster members before leaving the cluster. The first reason for that is to maintain the cluster structure even if the current $\mathrm{CH}$ leaves. The second reason is to avoid using the re-clustering algorithm and thus no re-clustering overhead is generated when the $\mathrm{CH}$ leaves the cluster.

Joining a cluster: The cluster head periodically broadcasts an ITJ (Invite-To-Join) message to its one-hop neighbors. Once a US or CHC vehicle receives an ITJ message, and if it wishes to join the cluster, it will send an RTJ (Request-To-Join) message including the vehicles ID, old_position, current_position, speed. When the $\mathrm{CH}$ receives the RTJ message, it will calculate the angle between its velocity vector and that of the requesting vehicle, and if the angle is less than $\phi$, the $\mathrm{CH}$ sends an acknowledgment (ACK) including its ID number. After the reception of the ACK, the corresponding vehicle becomes a $\mathrm{CM}$ of this cluster.

Leaving a cluster: A vehicle remains in the CM state as long as it receives an ITJ and has an acute angle with its cluster head. As shown in Figure 5, when a cluster member CM1 leaves its cluster, it will create an obtuse angle with its cluster head $\mathrm{CH} 1$. At instant $t+k$, the cluster head removes a CM1 from its cluster members list if the angle between their velocity vectors is greater than $\phi$.

Clusters merging When two or more $\mathrm{CHs}$ are moving in the same direction with an acute angle, only one of them will 


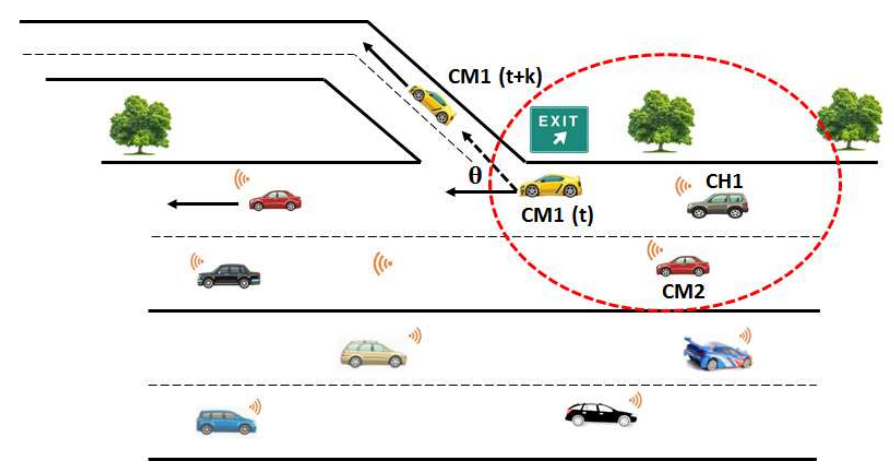

Fig. 5. Highway exit scenario.

keep its cluster head responsibility while the others will switch to a cluster member status. The selection of a cluster head for merging clusters is done based on the weight $\omega(i, t)$. In order to avoid false merges (when two clusters are merged and then they are separated just after merging), we restricted the merging to clusters having the same average speed.

\section{SIMULATION RESULTS AND PERFORMANCE EVALUATION}

This section presents ns- 2 simulation results to evaluate the performance of our proposed clustering algorithm ACA. The objectives of the evaluation are to: (1) evaluate the performance of ACA under different traffic conditions, (2) evaluate and compare the efficiency of ACA with other clustering protocols in the literature, (3) test the efficiency of ACA in reducing the number of states changed per vehicle.

\section{A. Simulation Scenarios}

We generated a realistic VANET environment by selecting a real highway area from a digital map which took into account lane directions. Figure 6 shows a metropolitan area from a map of San Jose (California) of size $3000 m \times 100 m$ exported from OpenStreetMap (OSM) and edited using Java OpenStreetMap Editor (JOSM). Then MOVE and SUMO [12] were used respectively to generate vehicular traffic scenarios and to simulate the area with vehicular traffic. To do that, we defined a vehicle flow which described a swarm of vehicles in each direction. The parameters of each vehicle flow consisted of the maximum number of vehicles, the starting road and destination of the flow, the time to start and end the flow. We assigned a random speed to each vehicle between $120 \mathrm{~km} / \mathrm{h}$ and $150 \mathrm{~km} / \mathrm{h}$. Then the traffic traces generated by SUMO were used in the $n s 2.34$ simulator. The simulation parameters used in our experiments are summarized in Table I.

TABLE I. Simulation parameters in ns-2

\begin{tabular}{cc}
\hline Parameter & Value/Protocol \\
\hline Simulation time & $80 \mathrm{~s}$ \\
Vehicle speed & $120-150 \mathrm{~km} / \mathrm{h}$ \\
Propagation model & Two Ray Ground \\
Medium Capacity & $6 \mathrm{Mbps}$ \\
Transmission range & $310 \mathrm{~m}$ \\
Transport Layer & UDP \\
CBR Packet Size & 512 bytes \\
Vehicle density $(\sigma)$ & 204080120160200240280300 \\
\hline
\end{tabular}
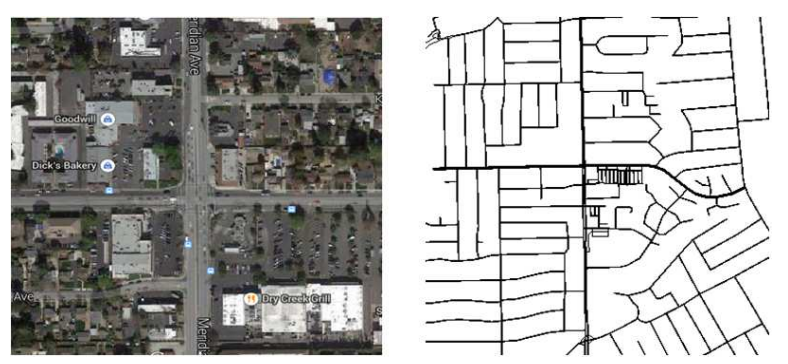

Fig. 6. San Jose (California) urban area captured from Google Maps (left) and exported to a VANET network topology by using MOVE/SUMO (right)

\section{B. Performance evaluations}

In this section, we evaluate and compare the performance of ACA with other clustering protocols proposed in the literature, namely AWCP [1], CPM [4] and HD [6]. We use the set of parameters found using the NSGA-II approach, see [11]. We simulate several scenarios by varying the vehicle density from 20 to 300 vehicles in the whole network. Figure 7 shows the cluster lifetime for the algorithms with different vehicle densities $(\sigma)$. This figure clearly shows that the cluster lifetime increases as the number of vehicles increases. We can see that ACA achieves a considerably longer cluster lifetime than CPM and HD. For instance, for a high density (when $\sigma=300$ ), the cluster lifetime is increased by $36.9 \%$ and $50.68 \%$ respectively. These results can be explained by the fact that, in ACA, only vehicles that are moving in the same direction with an acute angle can form a cluster and thus, the CMs will be associated with their $\mathrm{CHs}$ for a longer period of time. We can also note that CPM outperforms HD, because the CPM protocol forms clusters based on the mobility direction.

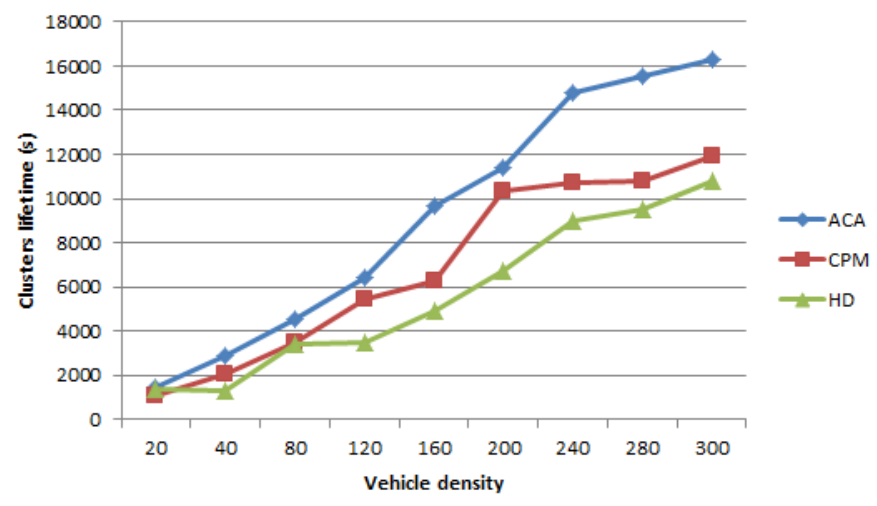

Fig. 7. Cluster lifetime under various traffic densities.

Figures 8 and 9 show the number of state transitions for each vehicle during the simulation for the scenarios where $\sigma$ equals 120 and 200, respectively. We can note from these two figures that ACA generates the lowest number of transitions. For instance, the vehicle of ID 70 in Figure 8 maintains its state throughout the simulation time when ACA is used, whereas it changes more than once when CPM or HD are used. These results are due to the fact that ACA avoids the problem of merging multiple clusters into a single cluster at road junctions.

In order to highlight the efficiency of ACA algorithm, 


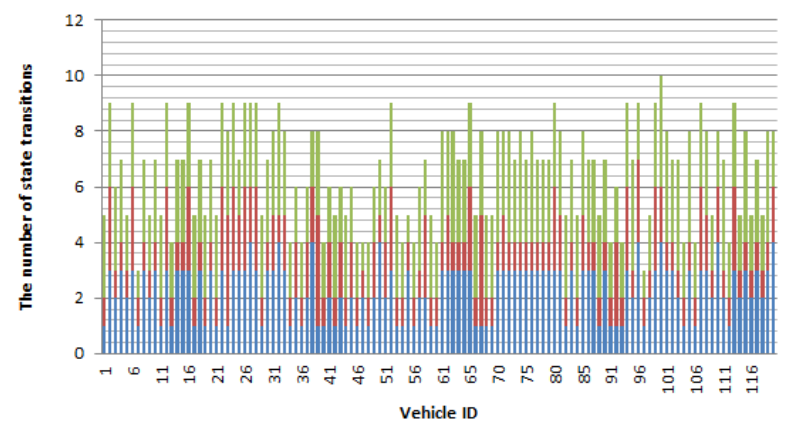

Fig. 8. The number of vehicle state transitions for $\sigma=120$.

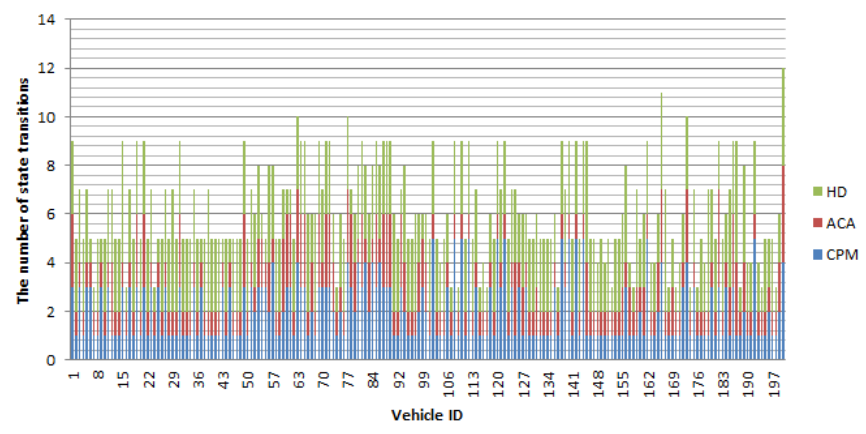

Fig. 9. The number of vehicle state transitions for $\sigma=200$.

we evaluate and compare it with the AWCP protocol in VANET scenarios where vehicles without maps are present. Figure 10 shows the Average Cluster Lifetime (ACL) for ACA and AWCP. These protocols are evaluated when we vary the number of vehicles which are not equipped with a digital map device between $20 \%, 40 \%$ and $50 \%$. As ACA is an anglebased clustering algorithm, the presence of vehicles that do not have map does not influence its performance. Moreover, when all the vehicles in the network have a map, the ACA and AWCP protocols have almost the same average cluster lifetime. However, we can note that the ACL metric decreases for AWCP as the number of vehicles that have no map increases. These results can be explained by the fact that, each mapunequipped vehicle that is in the $\mathrm{US}^{1}$ state joins any cluster without taking into account any road ID.

\section{CONCLUSiON}

The design of a stable clustering algorithm becomes a difficult task in VANETs when there are many road segments and intersections. In this paper, we propose an Angle-based Clustering Algorithm, named ACA, in which the velocity vector angle between the vehicles participating in the cluster head election process is used as a metric to improve cluster stability in VANETs. The simulation results show that ACA clearly improves the clustering performance in VANETs in terms of cluster lifetime. As future work, we plan to design a cross-layer architecture combining a Medium Access Control (MAC) protocol and a clustering scheme to improve channel access efficiency in VANETs where cluster head would be

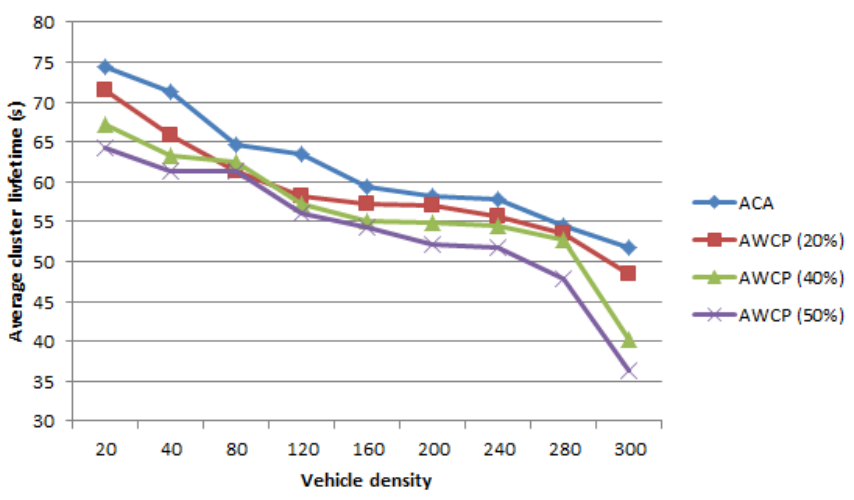

Fig. 10. Average Cluster lifetime under various traffic densities.

responsible for assigning bandwidth to all the members of its cluster.

\section{REFERENCES}

[1] M. Hadded, P. Muhlethaler, R. Zagrouba, A. Laouit, and L. A. Saidanel, "Using road ids to enhance clustering in vehicular ad hoc networks," in IEEE CEC, Dubrovnik, Croatia, Aug. 2015, pp. 285-290.

[2] Z. Y. Rawashdeh and S. M. Mahmud, "A novel algorithm to form stable clusters in vehicular ad hoc networks on highways," EURASIP Journal on Wireless Communications and Networking, Jan. 2012.

[3] M. M. C. Morales, C. S. Hong, and Y. C. Bang, "An adaptable mobilityaware clustering algorithm in vehicular networks," in APNOMS, Taipei, Taiwan, Sep. 2011, pp. 1-6.

[4] S. C. Lo, Y. J. Lin, and J. S. Gao, "A multi-head clustering algorithm in vehicular ad hoc networks," International Journal of Computer Theory and Engineering, vol. 5, no. 2, pp. 242-247, Apr. 2013.

[5] M. Gerla and J. C. Tsai, "Multicluster, mobile, multimedia radio network," Wireless Networks, vol. 1, no. 3, pp. 255-265, 1995.

[6] A. Ramalingam, S. Subramani, and K. Perumalsamy, "Associativity based cluster formation and cluster management in ad hoc networks," in IEEE HiPC, Bangalore, India, Dec. 2002, pp. 1-6.

[7] M. Chatterjee, S. K. Das, and D. Turgut, "A weighted clustering algorithm for mobile ad hoc networks," Cluster Computing, vol. 5, no. 2, pp. 193-204, Apr. 2002.

[8] P. Basu, N. Khan, and T. Littl, "A mobility based metric for clustering in mobile ad hoc networks," in ICDCS Workshop, Arizona, Apr. 2001, pp. 413-418.

[9] V. Naumov and T. Gross, "Connectivity-aware routing (car) in vehicular ad-hoc networks," in IEEE International Conference on Computer Communications, Anchorage, Alaska, May 2007.

[10] D. Tian, K. Shafiee, and V. C. Leung, "Position-based directional vehicular routing," in Global Telecommunications Conference, 2009. GLOBECOM 2009. IEEE, December, hawaii, USA, May 2009, pp. 16.

[11] M. Hadded, R. Zagrouba, A. Laouiti, P. Muhlethaler, and L. A. Saidane, "A multi-objective genetic algorithm-based adaptive weighted clustering protocol in vanet," in IEEE CEC, Sendai, Japan, May 2015, pp. 9941002.

[12] F. Karnadi, Z. Mo, and K. chan Lan, "Rapid generation of realistic mobility models for vanet," in IEEE WCNC, Hong Kong, China, Mar. 2007, pp. 2506-2511.

\footnotetext{
${ }^{1}$ Undecided State
} 